\title{
Development of Chinese Versions of Quality of Life in Late-Stage Dementia and Cognitive Tests for Severe Dementia
}

\author{
Suet-Lai Leung ${ }^{a, b} \quad$ Hiroyuki Tanaka ${ }^{c}$ Timothy C.Y. Kwok ${ }^{b, d}$ \\ aSchool of Medical and Health Sciences, Tung Wah College, Hong Kong, Hong Kong; \\ ${ }^{b}$ Department of Medicine and Therapeutics, The Chinese University of Hong Kong, Hong \\ Kong, Hong Kong; ' ${ }^{\circ}$ Graduate School of Comprehensive Rehabilitation, Osaka Prefecture \\ University, Osaka, Japan; dJockey Club Centre for Positive Ageing, Hong Kong, Hong Kong
}

\section{Keywords}

Chinese older adults · Cognitive Test for Severe Dementia - Quality of Life in Late-Stage

Dementia scale $\cdot$ Severe dementia

\begin{abstract}
Introduction: Valid assessments of quality of life (QoL) and cognition are important in caring for individuals with severe dementia; there is an urgent need for validated assessment tools for specific populations. This study aimed to develop and validate Chinese versions of the Quality of Life in Late-Stage Dementia (QUALID-C) scale and the Cognitive Test for Severe Dementia (CTSD-C) for Chinese older adults. Methods: This was a cross-sectional validation study comprised of 93 Chinese older adults with severe dementia recruited from 6 residential homes. The content and cultural validity of the QUALID-C and CTSD-C were evaluated by a 7-member expert panel, and interrater reliability, test-retest reliability, internal consistency, concurrent validity, and factorial structure were examined. Results: The QUALID-C showed acceptable internal consistency (Cronbach $\alpha=0.65$ ), good interrater reliability (intraclass correlation coefficient $[\mathrm{ICC}]=0.99$ ), and good test-retest reliability $(\mathrm{ICC}=0.96)$. Principal component analysis yielded 3 factors; the items loaded on the factors were comparable to those in previous studies and suggested the scale's multidimensionality to measure QoL. The CTSD$C$ showed satisfactory internal consistency (Cronbach $\alpha=0.862$ ), good interrater reliability $(I C C=0.99)$, and good test-retest reliability $(I C C=0.958)$. Principal component analysis yielded 3 factors; the items loaded on factors 1 and 2 resembled the items of the automatic response and attentional control factors of the original study. Conclusion: The QUALID-C and the CTSD-C are reliable and valid scales to measure the QoL and cognitive functions of Chinese older adults with severe dementia. These assessments can be utilized to evaluate the effectiveness of treatment and future research work.




\section{Introduction}

Rapidly ageing populations and an increasing dementia prevalence are global concerns. In Hong Kong, the population aged 60 years or older was reported to have increased to 1,351,000 (19.2\%) in 2011 and is projected to be nearly 3 million by 2039 [1]. Further, the number of people older than 60 years with dementia is predicted to increase from 103,433 in 2009 to 332,688 in 2039 [2].

Epidemiological studies suggest that approximately $20-40 \%$ of people with dementia are in the severe stage $[3,4]$. Although the average survival time of people older than 65 years ranges from 4 to 8 years after being diagnosed with Alzheimer's dementia, some patients may live up to 20 years. Care costs also increase dramatically from the mild stage to the severe stage, and the latter stage may account for $70-80 \%$ of the total care costs [5].

The significant prevalence, marked functional impairment, care burden, and cost related to severe dementia make it a vital healthcare issue [5]. Reliable and valid assessments for quality of life (QoL) and cognitive function are essential for clinical utility and evidence-based practice for people with severe dementia.

QoL is a multifaceted concept that covers subjective experiences and objective criteria [6-9]. In people with dementia, QoL encompasses cognitive function, psychosocial wellbeing, social interaction, and activities of daily living [10]. Severe loss of cognitive and language functions poses difficulties for assessing subjective experiences in late-stage dementia [11]. Most existing dementia-specific QoL scales are designed for mild to moderate stages and may be inapplicable to people with severe dementia [12].

The Quality of Life in Late-Stage Dementia (QUALID) scale was specifically developed to assess QoL in severe dementia [13]. It takes 5-10 min to complete and is rated based on observable behaviours of people with severe dementia. It has good psychometric properties $[13,14]$ and has been translated into different languages and validated in several countries [15-18].

Cognitive tests for severe dementia should be based on performance and assess simple reactions $[3,19]$, cover different cognitive domains, focus on residual abilities, and be sensitive enough to capture cognitive changes in people with severe dementia. Furthermore, the administration time should be short, so that it is tolerated by people with severe dementia [3].

The Severe Impairment Battery (SIB) takes $30 \mathrm{~min}$ to complete and may not be feasible for people with severe dementia [20,21]. The SIB short version takes 10-15 min and requires specialized training and equipment [22]. Another cognitive test, i.e., the Severe Mini-Mental Stage Examination, can be administrated in 5 min without special equipment; however, it may not be sensitive enough to measure cognitive changes [23]. The Cognitive Test for Severe Dementia (CTSD) takes approximately $10 \mathrm{~min}$ to administer and does not require special equipment [24]. It has been shown to significantly correlate with other cognitive assessments and can reliably capture cognitive changes in severe dementia over time [18, 24].

Although having valid outcome measures when caring for people with severe dementia is of increasing importance, no such instruments are available in Hong Kong. Therefore, an urgent need exists to create reliable and valid tools locally. The present study aimed to develop and examine the psychometric properties and factorial structures of the Chinese versions of the QUALID (QUALID-C) and the CTSD (CTSD-C).

\section{Materials and Methods}

\section{Participants}

This was a cross-sectional study. Different types of residential homes from the list of "residential care homes for older people" of the Social Welfare Department were invited to collaborate 
and recruit eligible participants by convenience sampling. Participants were from 6 different residential homes (i.e., 2 private, 2 self-financed, and 2 subsidized homes) located in 3 territories. Eligible participants: (1) were aged 65 years or older, (2) had a documented medical diagnosis of a major neurocognitive disorder (dementia) according to the Diagnostic and Statistical Manual of Mental Disorders V [25], and (3) had severe dementia, with a Chinese Clinical Dementia Rating (CCDR) of 3 [26, 27]. Exclusion criteria were: (1) severely impaired consciousness, (2) blindness, (3) deafness, or (4) diagnosis of bipolar affective disorder or schizophrenia.

\section{Procedure}

After receiving permission from the original authors of the QUALID and the CTSD, 2 independent occupational therapists (OT) with related work experience performed double translations. They were both fluent in Chinese and English. The OT was blind to the original versions when performing backward translations. Backward translations of the QUALID-C and the CTSD-C were sent to the original authors for verification. In forward translation of the CTSD, 1 of 3 terms, i.e., "cherry blossom" in question 4, was changed to "peach blossom," due to cultural relevancy, and the original author agreed to the change. The content validity of the Chinese versions of both instruments was examined by a 7-member expert panel of geriatric psychiatrists, OT, and a social worker with more than 20 years of relevant work experience. These experts rated the relevance and representativeness of the QUALID-C and the CTSD-C for assessing the cognitive function and QoL of Chinese older adults with severe dementia via self-report questionnaires. The panel members' comments, suggestions, and ratings justifications were recorded.

Four OT (raters), including the principal investigator, went to each residential home to collect data and administer all instruments. Participant demographic and clinical data were retrieved by caseworkers from case files at each residential home. Participants were assessed using the CTSD-C and the Chinese (Cantonese) version of the Hierarchic Dementia Scale (CV-HDS) $[28,29]$ to examine the concurrent validity of the CTSD-C participants' caregivers were interviewed to complete the QUALID-C and the Chinese version of the Quality of Life in Alzheimer's Disease (CQOL-AD) scale [30] to evaluate the concurrent validity of the QUALID-C. To examine the interrater reliability of the CTSD-C and the QUALID-C, participants and their caregivers were assessed by one OT and rated by another OT simultaneously. To evaluate both assessments' test-retest reliability, participants and caregivers were reassessed within 10 days after the first administration by the same rater. The data collection period was between January and July 2019.

\section{Instruments}

The QUALID [13] is a proxy-rated scale comprising 11 items on observable moods and behaviours. Answers are rated on a 5-point scale, based on occurrences in the prior week. The maximum score is 55; lower scores indicate a better QoL [13].

The CTSD [24] comprises 13 items across 7 cognitive domains, i.e., orientation, memory, language, visuospatial, praxis and frontal function, and social interaction. The maximum score is 30; higher scores indicate better cognitive function [24].

The CQOL-AD scale [30] comprises 13 items measuring aspects of mental and physical health, interpersonal relationships, and overall QoL. The maximum total score is 52; higher scores indicate a better QoL. Patient and caregiver versions are available [30]. This study used the caregiver version, as participants had severe-stage dementia.

The CV-HDS is a cognitive assessment validated in Hong Kong with satisfactory psychometric properties. It comprises 20 questions and 9 cognitive areas, i.e., attention, orientation, memory, perception, calculation, language, praxis, and spatial and prefrontal function. The maximum total score is 200; higher scores indicate better cognitive function [28].

\section{Karger'}




\section{Data Analysis}

Statistical analyses were performed using IBM SPSS version 23.0. Descriptive statistics were used to analyse the expert panel's ratings in evaluating content validity. Any item rated as "good" or "very good" by $70 \%$ of the experts was regarded as relevant and representative, while items below 70\% were reviewed by the panel for further revision. As $70 \%$ agreement represented the majority opinion on the panel, it was considered safe to accept the items [29].

Intraclass correlation coefficients (ICC) were used to calculate the test-retest and interrater reliabilities of the QUALID-C and the CTSD-C [31]. ICC ranging from 0.80 to 1.00 were regarded as perfect, those ranging from 0.60 to 0.79 were almost perfect, and those ranging from 0.40 to 0.59 were adequate; coefficients $<0.40$ were inadequate [32]. Cronbach $\alpha$ was used to examine the 2 scales' internal consistency. Pearson correlation coefficients were used to evaluate concurrent validity between the QUALID-C and the CQOL-AD and the CTSD-C and the HDS-CV. Principal component analysis (PCA) was used to examine both instruments' factor structures. The number of components was based on the criteria of an eigenvalue above 1 and factor loading $>0.4$ [33].

\section{Results}

Translation and Content Validity of the QUALID-C and CTSD-C

All expert panel members rated the overall QUALID-C as "very good" or "good" for local application. Additionally, 74-100\% of the panel members rated all 11 items as having "very good" or "good" relevance and representativeness. Six of the 7 panel members rated the overall CTSD-C as "very good" or "good" when applied locally. Further, 74-100\% of the panel members rated all 13 items as having "very good" or "good" relevance and representativeness.

\section{Participants and Score Distribution}

The mean age \pm SD of the 93 participants was $88.01 \pm 7.9$ years, ranging from 65 to 104 years; 84 were women (90\%). The mean number of years of education was $2.75 \pm 4.4$, ranging from 0 to 16 years. All of the participants scored 3 on the CCDR. Of the participants, $64.5 \%$ were diagnosed with Alzheimer disease $(n=60), 10.8 \%$ with vascular dementia $(n=10)$, and $24.7 \%$ with nonspecific dementia $(n=23)$. The distribution of QUALID-C scores was slightly skewed left; the distribution of CTSD-C scores was slightly skewed right (Table 1).

\section{Psychometric Properties of the QUALID-C}

The Cronbach $\alpha$ coefficient estimated the internal consistency of the QUALID-C for all of the participants to be 0.650 . The item-total correction was 0.2 or higher for all items except "appears calm and comfortable" ( $\alpha=0.157)$ and "enjoys eating" $(\alpha=0.114)$. The $\alpha$ value did not increase significantly with the removal of any item from the analysis. Concurrent validity between QUALID-C and CQOL-AD total scores was significant (Pearson coefficient $=-0.525$; $p<0.001)$. The ICC between raters for total scores and individual items were significant. The test-retest reliability for total scores as measured by the ICC was significant. The ICC was significant for all items except "appears sad" and "cries" (Table 2).

\section{QUALID-C Factor Analysis}

PCA of the QUALID-Cyielded 3 factors accounting for $56.4 \%$ of the variability (Table 3 ). Factor 1 contained 4 items ("appears sad", "appears calm and comfortable", "verbalization suggests discomfort", and "being irritable and aggressive"), factor 2 contained 4 items ("smiles", "enjoys touch/being touched", "enjoys eating", and "enjoys social interaction"), and factor 3 contained 3 items ("cries", "facial expression of discomfort", and "appears physically uncomfortable").

\section{Karger'}


Dementia and Geriatric Cognitive Disorders Extra

Table 1. Total score distribution of the QUALID-C and the CTSD-C
Dement Geriatr Cogn Disord Extra 2020;10:172-181

Table 2. Reliability of ICC

\begin{tabular}{lll}
\hline & $\begin{array}{l}\text { Interrater } \\
\text { reliability } \\
(n=63)\end{array}$ & $\begin{array}{l}\text { Test-retest } \\
\text { reliability } \\
(n=28)\end{array}$ \\
\hline Total score & $0.990^{* *}$ & $0.872^{* *}$ \\
Individual item & $0.991^{* *}$ & $0.584^{*}$ \\
Smiles & $0.986^{* *}$ & 0.437 \\
Appears sad & $0.951^{* *}$ & 0.436 \\
Cries & $0.996^{* *}$ & $0.803^{* *}$ \\
Facial expression of discomfort & $0.982^{* *}$ & $0.883^{* *}$ \\
Enjoys touch/being touched & $0.993^{* *}$ & $0.590^{*}$ \\
Appears calm and comfortable & $0.996^{* *}$ & $0.832^{* *}$ \\
Appears physically uncomfortable & $0.994^{* *}$ & $0.670^{*}$ \\
Verbalization suggests discomfort & $1.000^{* *}$ & $0.572^{*}$ \\
Being irritable and aggressive & $1.000^{* *}$ & $0.740^{*}$ \\
Enjoys eating & $0.964^{* *}$ & 0.399 \\
Enjoys social interaction & &
\end{tabular}

${ }^{\mathrm{a}}$ ICC based on a two-way random-effects model. ${ }^{\mathrm{b}}$ ICC based on a two-way mixed model. ${ }^{*} p<0.05 .{ }^{* *} p<$ 0.001 .

Table 3. Factor analysis of the QUALID-C (PCA with varimax rotation)

\begin{tabular}{lccc}
\hline \multirow{2}{*}{ Item } & \multicolumn{2}{l}{ Factor } & \\
\cline { 2 - 4 } & 1 & 2 & 3 \\
\hline Smiles & 0.088 & $\mathbf{0 . 6 5 2}$ & 0.285 \\
Appears sad & $\mathbf{0 . 5 9 5}$ & 0.132 & 0.053 \\
Cries & 0.122 & 0.018 & $\mathbf{0 . 5 4 1}$ \\
Facial expression of discomfort & 0.549 & 0.093 & $\mathbf{0 . 5 6 4}$ \\
Enjoys touch/being touched & 0.327 & $\mathbf{0 . 7 0 8}$ & -0.372 \\
Appears calm and comfortable & $\mathbf{0 . 5 9 2}$ & -0.392 & 0.129 \\
Appears physically uncomfortable & 0.255 & -0.063 & $\mathbf{0 . 7 4 8}$ \\
Verbalization suggests discomfort & $\mathbf{0 . 7 1 5}$ & 0.064 & 0.276 \\
Being irritable and aggressive & $\mathbf{0 . 7 4 1}$ & 0.074 & 0.163 \\
Enjoys eating & -0.257 & $\mathbf{0 . 6 8 6}$ & 0.268 \\
Enjoys social interaction & 0.138 & $\mathbf{0 . 7 6 9}$ & -0.289 \\
Cronbach $\alpha$ coefficient & 0.67 & 0.65 & 0.57 \\
Eigenvalue & 2.88 & 2.21 & 1.11 \\
Explained variance, \% & 26.20 & 20.10 & 10.10 \\
\hline
\end{tabular}

Bold type denotes significance. PCA, principal component analysis; QUALID-C, Chinese Version of Quality of Life in Late-Stage Dementia. 
Table 4. Reliability of the CTSD-C by ICC

\begin{tabular}{lll}
\hline & $\begin{array}{l}\text { Interrater } \\
\text { reliability } \\
(n=43)\end{array}$ & $\begin{array}{l}\text { Test-retest } \\
\text { reliability } \\
(n=34)\end{array}$ \\
\hline Total score & $0.996^{* *}$ & $0.964^{* *}$ \\
Individual item & $0.845^{* *}$ & $0.854^{* *}$ \\
Greeting & $0.841^{* *}$ & $0.825^{* *}$ \\
Giving one's name & $1.000^{* *}$ & $0.597^{* *}$ \\
Date of birth & $0.995^{* *}$ & $0.715^{* *}$ \\
Repetition & $1.000^{* *}$ & $0.704^{* *}$ \\
Naming objects & $1.000^{* *}$ & $0.813^{*}$ \\
Immediate memory & $1.000^{* *}$ & $0.543^{*}$ \\
Clock reading & $0.997^{* *}$ & $0.708^{* *}$ \\
Using items & $0.974^{* *}$ & $0.691^{* *}$ \\
Naming colours & $0.986^{* *}$ & $0.825^{* *}$ \\
Naming vegetables & $1.000^{* *}$ & $0.863^{* *}$ \\
Follow simple instructions & $0.974^{* *}$ & $0.974^{* *}$ \\
Copying a square & $1.000^{* *}$ & $0.876^{* *}$ \\
Enjoys social interaction &
\end{tabular}

a ICC based on a two-way random-effects model. ${ }^{\mathrm{b}}$ ICC based on a two-way mixed model. ${ }^{*} p<0.05 .{ }^{* *} p<0.001$.

Psychometric Properties of the CTSD-C

The internal consistency for all of the participants, estimated by Cronbach $\alpha$, was 0.862 . The $\alpha$ value of all individual items was between 0.317 and 0.764 . The concurrent validity between CTSD-C and CV-HDS total scores was significant (Pearson coefficient $=0.858 ; p<$ $0.001)$. The ICC between raters on total scores and all individual items were significant. The test-retest reliability for total scores and all individual items was also significant (Table 4).

\section{CTSD-C Factor Analysis}

PCA of the CTSD-C yielded 3 factors accounting for $65.13 \%$ of the variability (Table 5). Factor 1 contained 9 items ("greeting", "giving one's name", "repetition", "naming objects", "immediate memory", "using items", "naming colours", "naming vegetables", and "follow simple instructions"), factor 2 contained 3 items ("clock reading", "coping a square", and "writing one's own name"), and factor 3 contained the item "date of birth".

\section{Discussion}

This study examined the psychometric properties and factorial structure of the QUALID-C and the CTSD-C. Consistent with previous findings, the results showed that both of the instruments had good reliability and validity, thus indicating the Chinese translations of both scales were accurate.

Scores of both the QUALID-C and CTSD-C showed a relatively normal distribution. The proportions of participants who scored at the minimum or maximum were low, suggesting that the scales did not have floor or ceiling effects [33].

The internal consistency of the QUALID-C was moderate in this study, which was slightly lower than the results for other versions of the QUALID [13, 16-18]. In this study, the items "enjoys eating" and "appears calm and comfortable" showed a low item-total correlation; 
Table 5. Factor analysis of the CTSD-C (PCA with promax rotation)
Leung et al.: Chinese Versions of Dementia Scales

\begin{tabular}{lcrr}
\hline Item & \multicolumn{2}{l}{ Factor } & \\
\cline { 2 - 4 } & \multicolumn{2}{l}{2} & \multicolumn{2}{l}{3} \\
\hline Greeting & $\mathbf{0 . 6 6 5}$ & 0.159 & 0.010 \\
Giving one's name & $\mathbf{0 . 8 4 6}$ & 0.381 & -0.109 \\
Date of birth & 0.172 & 0.243 & $\mathbf{0 . 8 4 3}$ \\
Repetition & $\mathbf{0 . 7 6 4}$ & 0.473 & 0.083 \\
Naming objects & $\mathbf{0 . 7 7 3}$ & 0.544 & -0.205 \\
Immediate memory & $\mathbf{0 . 6 1 3}$ & 0.575 & 0.288 \\
Clock reading & 0.501 & $\mathbf{0 . 7 0 8}$ & -0.406 \\
Using items & $\mathbf{0 . 8 4 3}$ & 0.465 & 0.079 \\
Naming colours & $\mathbf{0 . 6 6 3}$ & 0.531 & -0.389 \\
Naming vegetables & $\mathbf{0 . 7 1 9}$ & 0.642 & 0.169 \\
Follow simple instructions & $\mathbf{0 . 7 9 2}$ & 0.320 & 0.002 \\
Copying a square & 0.302 & $\mathbf{0 . 8 0 9}$ & 0.105 \\
Writing one's own name & 0.295 & $\mathbf{0 . 7 6 8}$ & 0.196 \\
Cronbach $\alpha$ coefficient & 0.87 & 0.72 & 0.88 \\
Eigenvalue & 5.74 & 1.56 & 1.17 \\
Explained variance, \% & 44.17 & 11.97 & 8.99 \\
\hline
\end{tabular}

Bold type denotes significance. PCA, principal component analysis; CTSD-C, Chinese version of Cognitive Test for Severe Dementia.

"enjoys eating," "cries," and "being irritable and aggressive" have been shown to have a low item-total correlations in previous studies $[13,17,34]$. The variations reported in different studies might be due to the different study populations.

Consistent with previous findings, the QUALID-C was shown to have good interrater and test-retest reliability for total scores. Concurrent validity between the QUALID-C and the CQOL-AD was moderate, as they were originally designed for different dementia severity levels.

PCA of the QUALID-C resulted in 3 factors with acceptable Cronbach $\alpha$ coefficients, suggesting the scale measures various QoL domains. The items loaded on 3 factors, and the total explained variability in this study was similar to the findings of Spanish and Norwegian studies $[15,17,35]$. In the present study, factor 2 included the same items as the factor "social interaction" in the Spanish study [17] and the factors "comfort" and "well-being" in 2 Norwegian studies [15, 35].

The CTSD-C showed good internal consistency and good interrater and test-retest reliability, in line with the original study's results $[18,24]$. Additionally, the CTSD-C was significantly correlated with the HDS-CV.

PCA of the CTSD-C resulted in 3 factors with acceptable Cronbach $\alpha$ coefficients. Items included in factor 1 were categorized as "low difficulty" in comparison to the items of factor $2[18,36]$. These items required fewer attentional resources, i.e., the "automatic response" factor, and are expected to deteriorate in late-stage dementia. The findings of this study were consistent with previous observations. Autobiographical memory included recall or reorganization of participants' own names, comprehension of simple commands, naming primary colours, and repetition, which tend to be well-preserved until the later stage of dementia $[18,36]$. Items loaded on factor 2 were categorized as "controlled information processing" and regarded as relatively difficult items. Item 3, i.e., "date of birth," emerged as a new factor in this study, and $78.5 \%$ of the participants failed to answer this question. It may be that recalling one's date of birth involves semantic memory, which 
declines in the early stage of dementia. Moreover, Chinese older adults have a unique culture and traditionally mark their age by the lunar calendar. Many older adults do not know their exact date of birth and only have their birth year on their Hong Kong identity cards.

Factors 1 and 2 identified in this study resembled the 2 factors "automatic response" and "attentional control" reported in the original study [18]. The item "clock reading" was categorized under automatic response in the original study [18] but categorized as "attentional control" in this study and the study conducted by Choe et al. [37]. The differences reported may be due to differences in the recruited samples, such as a larger sample size $(n=232)$, with participants having a higher education level (mean years of education $=10$ years) in the original study and participants recruited from hospital settings [18].

Some limitations should be addressed. The nonrandom selection of participants (rather small sample size) and the cross-sectional design may limit the results' generalizability. Nevertheless, the participants are recruited from different types of residential homes located in 3 territories, which enhances the representativeness. To the best of our knowledge, the QUALID-C and the CTSD-C are the first locally validated assessments to specifically measure QoL and cognitive function, respectively, for Chinese older people with severe dementia. The findings of the present study indicate that the QUALID-C and the CTSD-C are valid and reliable scales to evaluate QoL and cognitive function in Chinese older adults with severe dementia. They can be useful to evaluate the effectiveness of treatment in future research.

\section{Acknowledgement}

The authors thank all of the individuals who participated in this study together with their families and caregivers, as well as all residential homes for their assistance in the recruitment process. This research did not receive any specific grant from funding agencies in the public, commercial, or not-for-profit sector.

\section{Statement of Ethics}

This study was conducted with approval from the Tung Wah College Research Ethics Committee (reference No. RESC2018015) and in accordance with the Helsinki Declaration as revised in 2013. Caseworkers at each residential home screened the eligible participants and contacted their caregivers to explain this study and acquire informed consent. The Tung Wah College Ethics Committee reviewed and approved this study.

\section{Conflict of Interest Statement}

The authors have no conflict of interests to declare.

\section{Funding Sources}

There are no funding sources to declare. 


\section{Author Contributions}

S.-L.L. and Prof. Timothy C.Y. Kwok conceived and designed this study. S.-L.L. performed the data collection and analysis and drafted the initial version of this paper. All of the authors contributed to data interpretation. S.-L.L. and Prof. Timothy C.Y. Kwok approved the final version of this paper.

\section{References}

1 Census and Statistics Department of Hong Kong Special Administrative Region. Hong Kong population projections 2010-2039 [Internet]. [cited 2018 May 29]. Available from: http://www.statistics.gov.hk/publication/ statreport/population/B1120015042010XXXXB0100.pdf.

2 Yu R, Chau PH, McGhee SM, Cheung WL, Chan KC, Cheung SH, et al. Trends in prevalence and mortality of dementia in elderly Hong Kong population: projections, disease burden, and implications for long-term care. Int J Alzheimers Dis. 2012;2012:406852.

3 Boller F, Verny M, Hugonot-Diener L, Saxton J. Clinical features and assessment of severe dementia. A review. Eur J Neurol. 2002 Mar;9(2):125-36.

4 Hebert LE, Scherr PA, Bienias JL, Bennett DA, Evans DA. Alzheimer disease in the US population: prevalence estimates using the 2000 census. Arch Neurol. 2003 Aug;60(8):1119-22.

5 Herrmann N, Gauthier S, Lysy PG. Clinical practice guidelines for severe Alzheimer's disease. Alzheimers Dement. 2007 Oct;3(4):385-97.

6 Ettema TP, Dröes RM, de Lange J, Ooms ME, Mellenbergh GJ, Ribbe MW. The concept of quality of life in dementia in the different stages of the disease. Int Psychogeriatr. 2005 Sep;17(3):353-70.

7 Lawton MP. Quality of life in Alzheimer disease. Alzheimer Dis Assoc Disord. 1994;8 Suppl 3:138-50.

8 Lawton MP. Assessing quality of life in Alzheimer disease research. Alzheimer Dis Assoc Disord. 1997;11 Suppl 6:91-9.

9 Smit D, de Lange J, Willemse B, Twisk J, Pot AM. Activity involvement and quality of life of people at different stages of dementia in long term care facilities. Aging Ment Health. 2016;20(1):100-9.

10 Whitehouse PJ, Orgogozo JM, Becker RE, Gauthier S, Pontecorvo M, Erzigkeit H, et al. Quality-of-life assessment in dementia drug development. Position paper from the International Working Group on Harmonization of Dementia Drug Guidelines. Alzheimer Dis Assoc Disord. 1997;11 Suppl 3:56-60.

11 Rabins PV, Kasper JD. Measuring quality of life in dementia: conceptual and practical issues. Alzheimer Dis Assoc Disord. 1997;11 Suppl 6:100-4.

12 Hoe J, Hancock G, Livingston G, Woods B, Challis D, Orrell M. Changes in the quality of life of people with dementia living in care homes. Alzheimer Dis Assoc Disord. 2009 Jul-Sep;23(3):285-90.

13 Weiner MF, Martin-Cook K, Svetlik DA, Saine K, Foster B, Fontaine CS. The quality of life in late-stage dementia (QUALID) scale. J Am Med Dir Assoc. 2000 May-Jun;1(3):114-6.

14 Martin-Cook K, Hynan LS, Rice-Koch K, Svetlik DA, Weiner MF. Responsiveness of the quality of life in latestage dementia scale to psychotropic drug treatment in late-stage dementia. Dement Geriatr Cogn Disord. 2005;19(2-3):82-5.

15 Barca ML, Engedal K, Laks J, Selbæk G. Quality of life among elderly patients with dementia in institutions. Dement Geriatr Cogn Disord. 2011;31(6):435-42.

16 Falk H, Persson LO, Wijk H. A psychometric evaluation of a Swedish version of the Quality of Life in Late-Stage Dementia (QUALID) scale. Int Psychogeriatr. 2007 Dec;19(6):1040-50.

17 Garre-Olmo J, Planas-Pujol X, López-Pousa S, Weiner MF, Turon-Estrada A, Juvinyà D, et al. Cross-cultural adaptation and psychometric validation of a Spanish version of the Quality of Life in Late-Stage Dementia Scale. Qual Life Res. 2010 Apr;19(3):445-53.

18 Nagata Y, Tanaka H, Ishimaru D, Uematsu M, Naito Y, Nishikawa T. Development of the Japanese version of the Quality of Life in Late-stage Dementia Scale. Psychogeriatrics. 2018 Mar;18(2):106-12.

19 Ericsson I, Malmberg B, Langworth S, Haglund A, Almborg AH. KUD—a scale for clinical evaluation of moderateto-severe dementia. J Clin Nurs. 2011 Jun;20(11-12):1542-52.

20 Pippi M, Mecocci P, Saxton J, Bartorelli L, Pettenati C, Bonaiuto S, et al. Neuropsychological assessment of the severely impaired elderly patient: validation of the Italian short version of the Severe Impairment Battery (SIB) Gruppo di Studio sull'Invecchiamento Cerebrale della Società Italiana di Gerontologia e Geriatria. Aging (Milano). 1999 Aug;11(4):221-6.

21 Saxton J, Boller F. Cognitive functions in severe dementia. In: Burns A, Winblad B, editors. Severe dementia. Hoboken (NJ): John Wiley \& Sons; 2006. pp. 43-9.

22 de Jonghe JF, Wetzels RB, Mulders A, Zuidema SU, Koopmans RT. Validity of the Severe Impairment Battery Short Version. J Neurol Neurosurg Psychiatry. 2009 Sep;80(9):954-9.

23 Harrell LE, Marson D, Chatterjee A, Parrish JA. The Severe Mini-Mental State Examination: a new neuropsychologic instrument for the bedside assessment of severely impaired patients with Alzheimer disease. Alzheimer Dis Assoc Disord. 2000 Jul-Sep;14(3):168-75. 


\section{Geriatric Cognitive Disorders Extra}

24 Tanaka H, Nagata Y, Uematsu M, Takebayashi T, Hanada K, Inokawa M, et al. Development of the cognitive test for severe dementia. Dement Geriatr Cogn Disord. 2015;40(1-2):94-106.

25 American Psychiatric Association. Diagnostic and statistical manual of mental disorders. 5th ed. Washington (DC): American Psychiatric Association Publishing; 2013.

26 Hughes CP, Berg L, Danziger WL, Coben LA, Martin RL. A new clinical scale for the staging of dementia. Br J Psychiatry. 1982 Jun;140(6):566-72.

27 Lai KY, Lau LK, Ng L, Faulkner LW, Chung CC, Wong TK. Cross cultural validation: the Chinese version of the Clinical Dementia Rating scale. Asian J Nurs Stud. 2004;7(1):36-41.

28 Poon VW, Lam LC, Wong SY. Assessment of the severity of dementia: validity and reliability of the Chinese (Cantonese) version of the Hierarchic Dementia Scale (CV-HDS). Int J Geriatr Psychiatry. 2008 Sep;23(9):909-14.

29 Terwee CB, Bot SD, de Boer MR, van der Windt DA, Knol DL, Dekker J, et al. Quality criteria were proposed for measurement properties of health status questionnaires. J Clin Epidemiol. 2007 Jan;60(1):34-42.

30 Chan IW, Chu LW, Lee PW, Li SW, Yu KK. Effects of cognitive function and depressive mood on the quality of life in Chinese Alzheimer's disease patients in Hong Kong. Geriatr Gerontol Int. 2011 Jan;11(1):69-76.

31 Koo TK, Li MY. A guideline of selecting and reporting intraclass correlation coefficients for reliability research. J Chiropr Med. 2016 Jun;15(2):155-63.

32 Landis JR, Koch GG. The measurement of observer agreement for categorical data. Biometrics. 1977 Mar; 33(1):159-74

33 Munro BH. Statistical methods for health care research. Philadelphia (PA): Lippincott Williams \& Wilkins; 2000.

34 Røen I, Selbæk G, Kirkevold Ø, Engedal K, Lerdal A, Bergh S. The reliability and validity of the Norwegian version of the Quality of Life in Late-Stage Dementia scale. Dement Geriatr Cogn Disord. 2015;40(3-4):233-42.

35 Mjørud M, Kirkevold M, Røsvik J, Engedal K. Principal component analysis of the Norwegian version of the quality of life in late-stage dementia scale. Dement Geriatr Cogn Disord. 2014;37(5-6):265-75.

36 Jorm AF. Controlled and automatic information processing in senile dementia: a review. Psychol Med. 1986 Feb;16(1):77-88.

37 Choe JY, Youn JC, Park JH, Park IS, Jeong JW, Lee WH, et al. The Severe Cognitive Impairment Rating Scale-an instrument for the assessment of cognition in moderate to severe dementia patients. Dement Geriatr Cogn Disord. 2008;25(4):321-8. 